\title{
Evaluation of Guinea Pig Model for Ocular and Cervical Vestibular-Evoked Myogenic Potentials for Vestibular Function Test
}

Ting-Hua Yang, MD; Shing-Hwa Liu, PhD; Yi-Ho Young, MD

Objectives/Hypothesis: This study used airconducted sound (ACS) and bone-conducted vibration (BCV) stimuli in eliciting ocular vestibular-evoked myogenic potential (oVEMP) and cervical VEMP (cVEMP) in guinea pigs.

Study Design: Prospective study.

Methods: Ten guinea pigs were treated with gentamicin $(4 \mathrm{mg})$ on the left ear, whereas the right ear served as a control. One week after treatment, each animal underwent oVEMP and cVEMP tests using ACS and BCV modes in a randomized order, and was sacrificed for morphological study.

Results: Using ACS mode, oVEMPs were absent in all $10(100 \%)$ animals despite the stimulus intensity increased up to $120 \mathrm{~dB}$ pe SPL. Conversely, using BCV mode, oVEMPs were present on the left (lesion) eye, and absent on the right (control) eye in all (100\%) animals. For the cVEMPs via ACS mode, all right (control) necks had clear cVEMPs, and all (100\%) left (lesion) necks revealed absent cVEMPs. However, via BCV mode, all right (control) necks and six $(60 \%)$ left (lesion) necks showed clear cVEMPs. Morphological study demonstrated substantial loss of hair cells in the utricular and saccular macula.

Conclusions: The cVEMP test via ACS mode is specific for investigating the saccular disorder, whereas the oVEMP test via BCV mode is preferable for investigating the utricular disorders in humans. The guinea pig model is consistent with the findings of humans. Restated, appropriate animal models for cVEMP and oVEMP in guinea pigs are via ACS and BCV modes, respectively.

From the Department of Otolaryngology, National Taiwan University Hospital, Taipei, Taiwan (T-H.YANG, Y.-H.YOUNG); and the Institute of Toxicology (T.-H.YANG, S.-H.L.), College of Medicine, National Taiwan University, Taipei, Taiwan.

Editor's Note: This Manuscript was accepted for publication May 21, 2010.

This work was supported by National Taiwan University Hospital, Taipei, Taiwan, grant number: NTUH97-M-1009. The authors have no other funding, financial relationships, or conflicts of interest to disclose.

Send correspondence to Yi-Ho Young, MD, Department of Otolaryngology, National Taiwan University Hospital, 1, Chang-Te St., Taipei, Taiwan. E-mail: youngyh@ntu.edu.tw

DOI: 10.1002/lary.21056
Key Words: Air-conducted sound, bone-conducted vibration, cervical vestibular-evoked myogenic potential, ocular vestibular-evoked myogenic potential, guinea pigs.

Level of Evidence: 2c. Laryngoscope, 120:1910-1917, 2010

\section{INTRODUCTION}

Using air-conducted sound (ACS) or bone-conducted vibration (BCV) stimuli, vestibular-evoked myogenic potentials (VEMPs) have been successfully recorded on the tonically contracted cervical muscles, so-called cervical VEMPs (cVEMPs), and on the extraocular muscles, socalled ocular VEMPs (oVEMPs). ${ }^{1}$ The ACS-stimulated cVEMPs are believed to evaluate the ipsilateral sacculocollic reflex, which primarily originates from the saccule. ${ }^{2}$ In contrast, the BCV-stimulated oVEMPs assess the crossed vestibulo-ocular reflex (VOR) pathway, and irregular otolithic afferents are preferentially activated by BCV stimuli. ${ }^{3,4}$ In humans, the optimal stimuli for eliciting cVEMPs and oVEMPs are via ACS and BCV modes, respectively. ${ }^{5}$ Clinically, the ACS-cVEMPs provide an essential merit for site selectivity, whereas BCV-oVEMPs have been adopted for mass detection. ${ }^{6}$

In experimental animals, we have successfully recorded cVEMPs in alert guinea pigs using ACS mode, ${ }^{7}$ which set the stage to study the mechanism of the saccular disorders. ${ }^{8,9}$ Recently, an animal model of oVEMP using BCV mode has also been established at our laboratory. ${ }^{10}$ However, it is unclear whether ACS or BCV mode is the optimal way to induce the oVEMP or cVEMP in guinea pigs. Hence, this study used ACS and BCV modes in eliciting oVEMPs and cVEMPs in guinea pigs. Our final goal was to develop appropriate animal models for oVEMPs and cVEMPs.

\section{MATERIALS AND METHODS}

\section{Animal Preparation}

Ten Hartley-strain guinea pigs weighing 200 to 250 g were used in this study. Animals were anesthetized by an intraperitoneal injection of pentobarbital sodium $(35 \mathrm{mg} / \mathrm{kg})$. The left tympanic bulla was opened via postauricular incision to have 
access to the round window membrane under operating microscope. A total of $100 \mu \mathrm{L}$ of gentamicin solution (40 mg gentamicin sulfate dissolved in $1 \mathrm{~mL}$ sterile aqueous solution) was dropped over the left (lesion) round window membrane. Then, the bulla was closed with overlying muscles, and the incision wound was sutured. The animal was kept on the recumbent position with the left ear up to ensure a good contact between the gentamicin and the round window membrane until the animal awoke from the anesthesia. Approximately 6 hours were needed for the animals to recover from the anesthesia. Spontaneous nystagmus and animal behavior were checked. Thereafter, animals were housed at $23 \pm$ $2{ }^{\circ} \mathrm{C}$ and $55 \pm 5 \%$ humidity and fed a solid diet and tap water ad libitum. One week after surgery, each animal underwent oVEMP and CVEMP tests in a randomized order, using ACS mode on one day and BCV mode on another day, and then the animal was sacrificed for morphological study.

This study was institutional review board approved, and was conducted in accordance with the guideline for the care and use of laboratory animals by the Animal Research Committee at National Taiwan University, College of Medicine.

\section{ACS Mode}

Click stimuli ( $120 \mathrm{~dB}$ pe SPL, duration $=0.1 \mathrm{~ms}$ ) were delivered through a short tube inserted into the ear canal. During recording (Smart EP 3.90; Intelligent Hearing Systems, Miami, FL), electromyographic signals were amplified and band-pass filtered to 1 to $1000 \mathrm{~Hz}$. Each animal underwent serial tests, with initial stimulus intensity of $120 \mathrm{~dB}$ pe SPL, followed by $10 \mathrm{~dB}$ decrements until the absence of waveform. The stimulus intensity was then increased in steps of 5 dBSPL until the VEMP response reappeared, and the threshold was thus determined. The stimulation rate was $5 \mathrm{~Hz}$ and the analysis time for each response was $24 \mathrm{~ms}$, with 200 responses being averaged for each run. Monaural acoustic stimulation with ipsilateral recording was conducted for recording cVEMPs, whereas contralateral recording was for recording oVEMPs.

\section{BCV Mode}

Single square-wave stimuli of $0.6 \mathrm{~ms}$ in duration were transmitted to a handheld vibrator with $1.81 \mathrm{~kg}$ in weight, $9.6 \mathrm{~cm}$ in height, and $7.8 \mathrm{~cm}$ in diameter (V201 vibrator; Ling Dynamic Systems, Royston, England) for head vibration, which was driven by a custom amplifier. Drive voltage was adjusted and fixed to produce a peak force level (FL) of $139 \mathrm{~dB}(\mathrm{re} 1 \mu \mathrm{N})$ from the vibrator, as measured by an artificial mastoid (model 4930; Bruel \& Kjaer P/L, Naerum, Denmark). The operator held the vibrator by hand and supported most of its weight such that the pole tip delivered a repeatable tap on the midline frontal bone of a guinea pig. Bilateral recordings were performed in oVEMP and cVEMP tests. Stimulation rate was $5 \mathrm{~Hz}$, analysis time for each response was $24 \mathrm{~ms}$, and 30 responses were averaged for each run.

Each animal underwent a serial VEMP testing, with the peak stimulus intensity of $139 \mathrm{dBFL}$ initially, followed by a 5 dBFL step decrement, until the absence of the waveforms. The stimulus intensity was then increased in steps of $5 \mathrm{dBFL}$ until the VEMP response reappeared, and the threshold was thus determined.

\section{oVEMP Test}

The head and body of a guinea pig was restrained but leaving its four legs free. Each animal was fixed with its head in a prone position. Five subdermal needle electrodes $(13.0 \times$ 0.4 mm, ES43-838; Technomed Europe, Beek, The Netherlands) were employed. On each side, one active electrode was inserted vertically on the skin $5 \mathrm{~mm}$ below the inferior orbital rim into the inferior extraocular muscle without force. The electrode carefully avoided contact with the eyeball with a depth of 5-10 $\mathrm{mm}$ from the skin. Another two reference electrodes were inserted approximately $15 \mathrm{~mm}$ below the respective active ones. The ground electrode was placed on the parietal area at the midline. The initial negative-positive biphasic waveform termed peaks $\mathrm{nI}$ and $\mathrm{pI}$ were measured by their latencies and amplitude. ${ }^{10}$

\section{cVEMP Test}

A pair of active needle electrodes was fitted on both dorsal neck extensor muscles at the level of the third cervical vertebral bone (C3), while a reference electrode was placed on the occipital area at the midline. During the recording, each animal was fixed with its head elevated and neck extended in a prone position throughout the test. The positive-negative polarities of biphasic waveforms were termed waves I and II. The latencies of positive peak I, negative peak II, and peak-to-peak I-II amplitude were measured. ${ }^{7}$

\section{Morphological Study}

After deep anesthesia with pentobarbital $(50 \mathrm{mg} / \mathrm{kg})$ administered intraperitoneally, all guinea pigs were decapitated. The temporal bones were harvested after removing the soft tissue, and the tympanic bulla were opened widely for checking the status of the tympanic cavities. Then, the membranous labyrinth was dissected immediately from the temporal bones under operating microscope. After the overlying otoconia were removed by mild stream of phosphate-buffered saline (PBS), the utricle and saccule were isolated separately as a whole mount preparation. Both utricular and saccular preparations were fixed in $4 \%$ paraformaldehyde and $1 \%$ glutaraldehyde in PBS for 20 minutes at $20^{\circ} \mathrm{C}$, and then permeabilized with 5\% Triton X-100 in PBS for 30 minutes. The utricular and saccular preparations were subsequently stained with rhodamine phalloidin (1:100; Sigma-Aldrich, St. Louis, MO) in PBS for 1 hour. Phalloidin specifically binds to F-actin, which is abundant in the stereocilia and cuticular plate of the hair cells. When the fluorescent dye rhodamine conjugated with phalloidin, it labeled F-actin and emitted red fluorescence. After staining, explants were washed three times with PBS and mounted onto glass slides with Fluoromount (Molecular Probes, Carlsbad, CA). Finally, slides were examined via confocal microscopy (Zeiss LSM 510; Meta, Gottingen, Germany). The excitation wavelength was $561 \mathrm{~nm}$ and the emission wavelength was longpass filtered over $575 \mathrm{~nm}$.

\section{Statistical Methods}

The prevalence, defined as percentage of ears (eyes) in which a clear response was identified, among various stimulus intensities were compared by Cochran's $Q$ test. One-way analysis of variance (ANOVA) followed by Bonferroni-adjusted $t$ test were used to conduct multiple comparisons between stimulus intensity and the latencies or amplitude. The latencies and amplitudes between right and left sides were compared by Mann-Whitney $U$ test. The latencies and amplitudes between various modes were compared by paired $t$ test. A significant difference indicates $P<.05$.

\section{RESULTS}

\section{oVEMPs: ACS versus BCV Modes}

Using ACS stimulation on the right (control) ears, oVEMPs were absent on the left (healthy) eyes of all (100\%) gentamicin-treated animals despite the stimulus intensity 

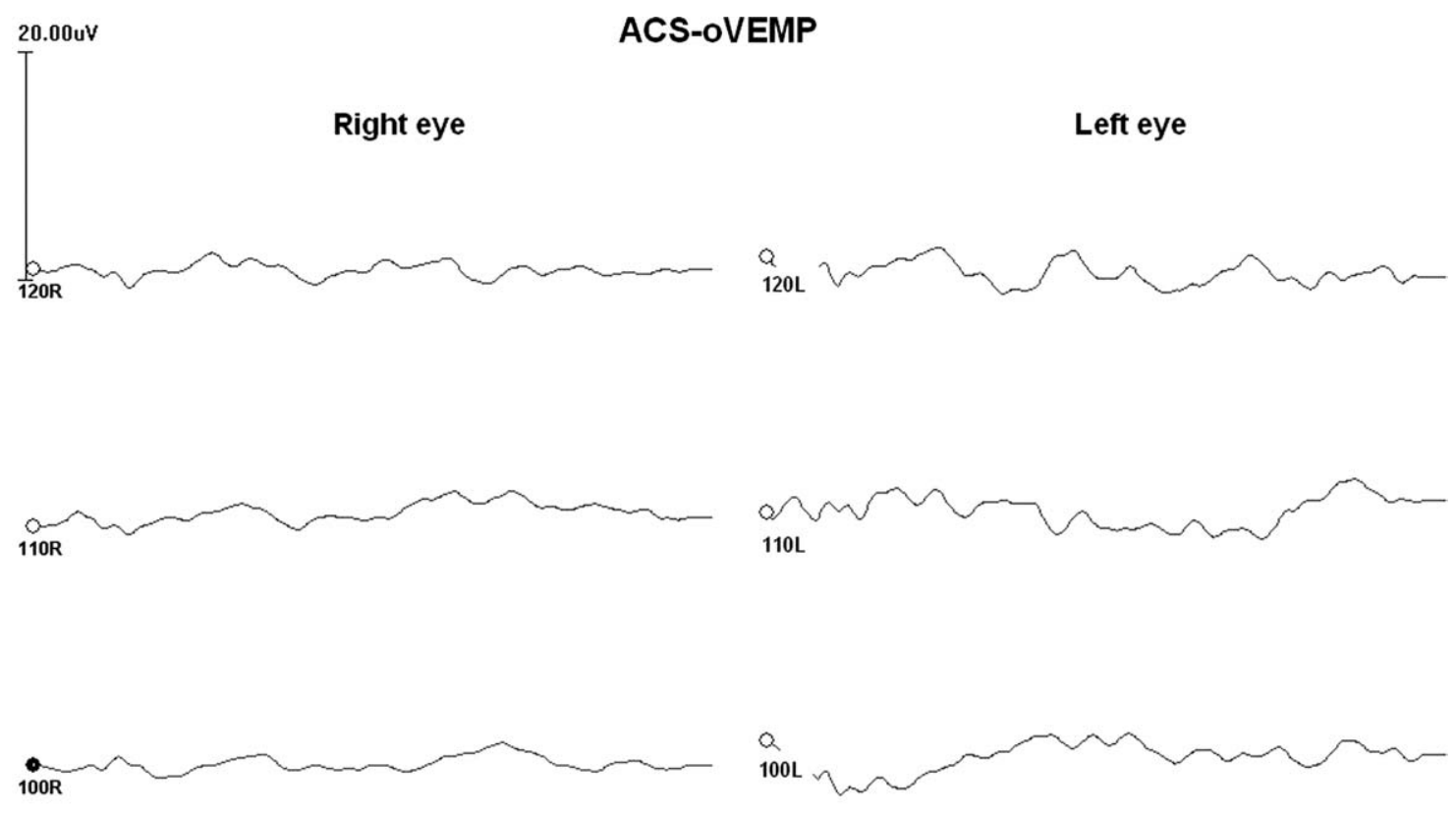

\begin{tabular}{lllllllll}
\hline 0 & 3 & 6 & 9 & 12 & 15 & 18 & 21 & $24 \mathrm{~ms}$
\end{tabular}

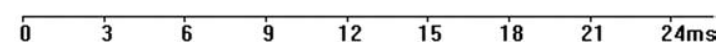

Fig. 1. Guinea pig treated with gentamicin (left), 1 week after treatment. Using air-conducted sound stimulation on the right ear, no ocular vestibular-evoked myogenic potential waveform is shown on the left eye $(120=120 \mathrm{~dB}$ pe SPL).

increased up to the maximum level of $120 \mathrm{~dB}$ pe SPL (Fig. 1). Conversely, using BCV stimuli, oVEMPs were absent on the right (control) eye, but clear oVEMPs were present on the left (lesion) eye in all (100\%) animals (Fig. 2).

The prevalence of BCV-oVEMPs with the peak stimulus intensity of 139,134 , and $129 \mathrm{dBFL}$ were $100 \%$,
$80 \%$, and $50 \%$, respectively, exhibiting a significant relationship between the prevalence and stimulus intensity $(P<.05$, Cochran $\mathrm{Q}$ test, Table I). The mean threshold of BCV-oVEMPs was $130 \pm 6 \mathrm{dBFL}$.

The mean latencies of $\mathrm{nI}$ of BCV-oVEMP with the intensity of 139,134 , and $129 \mathrm{dBFL}$ were $2.34 \pm 0.62$ (mean

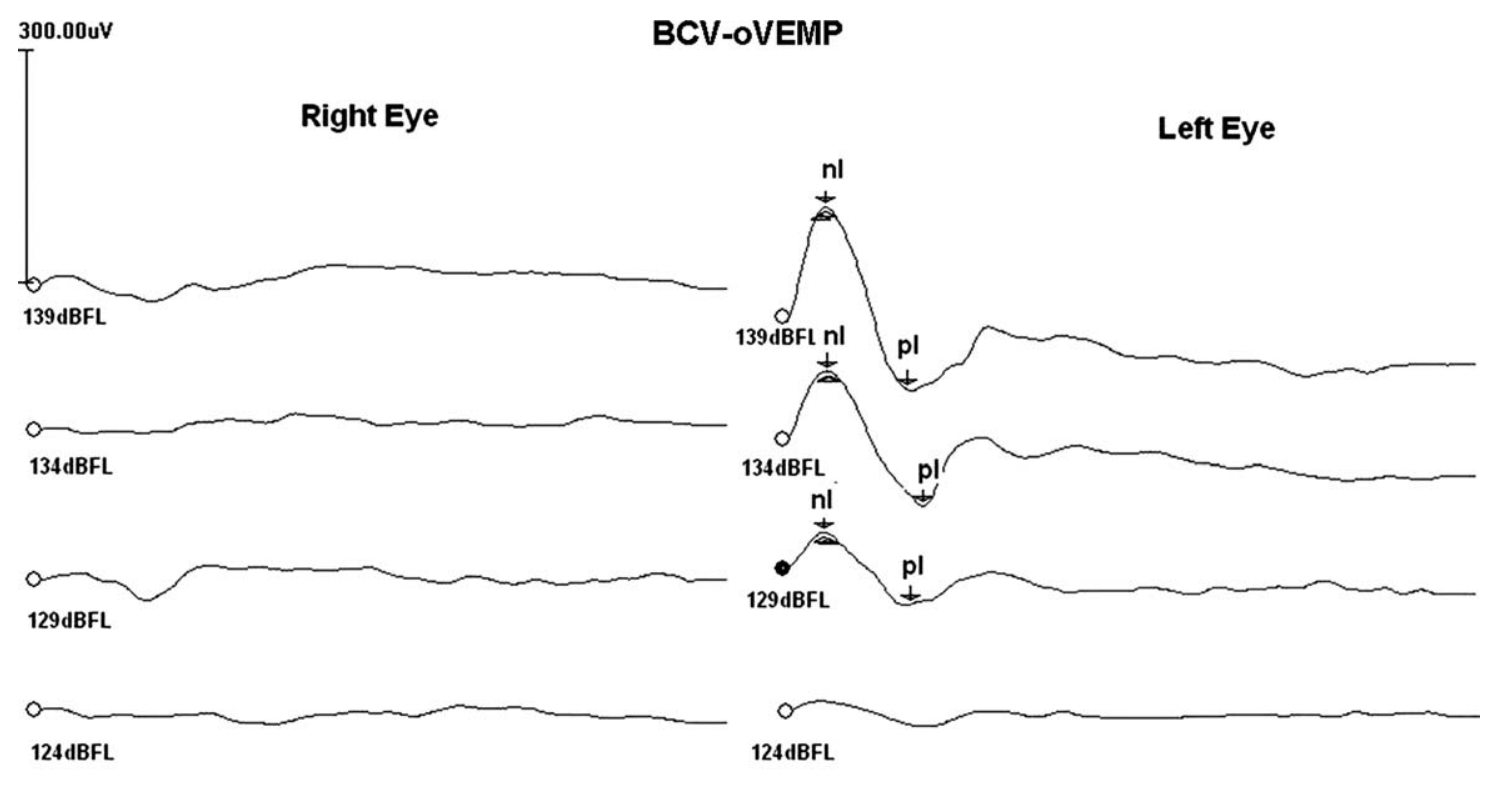

\begin{tabular}{lllllllll}
\hline 0 & 3 & 6 & $\dot{9}$ & 12 & 15 & 18 & 21 & $24 \mathrm{~ms}$
\end{tabular}

\begin{tabular}{lllllllll}
\hline 0 & 3 & 6 & 9 & 12 & 15 & 18 & 21 & $24 \mathrm{~ms}$
\end{tabular}

Fig. 2. Guinea pig treated with gentamicin (left), 1 week after treatment. Via bone-conducted vibration mode, ocular vestibular-evoked myogenic potentials are present on the left (lesion) eye, but not on the right (control) eye (139 dBFL $=139 \mathrm{~dB}$ force level). 
TABLE I.

Ocular Vestibular-Evoked Myogenic Potentials Using ACS Versus BCV Modes in Guinea Pigs.

\begin{tabular}{lcccccc}
\hline Stimulation Mode & Stimulus Intensity & No. Eyes & Prevalence, $\%$ & nl Latency, ms & pl Latency, ms & $\mathrm{nl}-\mathrm{pl}$ Amplitude, $\mu \mathrm{V}$ \\
\hline ACS & $120 \mathrm{~dB}$ pe SPL & 10 & 0 & 0 & 0 & 0 \\
BCV & $139 \mathrm{dBFL}$ & 10 & 100 & $2.34 \pm 0.62$ & $4.22 \pm 0.39$ & $100 \pm 62$ \\
& $134 \mathrm{dBFL}$ & 10 & 80 & $2.39 \pm 0.61$ & $4.11 \pm 0.39$ & $75 \pm 42^{\star}$ \\
& $129 \mathrm{dBFL}$ & 10 & 50 & $2.58 \pm 0.36$ & $4.0 \pm 0.29$ & $43 \pm 17^{\star}$ \\
& $\mathrm{p}$ value & & $<0.05^{\dagger}$ & $>0.05^{\ddagger}$ & $>0.05^{\ddagger}$ & $<0.05^{\S}$ \\
\hline \hline
\end{tabular}

Data are expressed as mean \pm standard deviation.

${ }^{\star} P<.05$, Bonferroni-adjusted $t$ test, as compared with the group of $139 \mathrm{dBFL}$.

${ }^{\dagger} P<.05$, Cochran $Q$ test.

${ }^{\ddagger} P>.05$.

$\S P<0.05$, one-way analysis of variance test.

$\mathrm{ACS}=$ air-conducted sound; BCV = bone-conducted vibration; $\mathrm{FL}=$ force level.

$\pm \mathrm{SD}), 2.39 \pm 0.61$, and $2.58 \pm 0.36 \mathrm{~ms}$, respectively, revealing nonsignificant difference among them $(P>.05$, one-way ANOVA test, Table I). Likewise, no significant difference existed in the mean latencies of pI among the three groups. Conversely, the mean nI-pI amplitude of BCV-oVEMP with the intensity of 139,134 , and $129 \mathrm{dBFL}$ were $100 \pm 62,75 \pm 42$, and $43 \pm 17 \mu \mathrm{V}$, respectively, showing a significant relationship between the stimulus intensity and nI-pI amplitude $(P<.05$, Table I).

\section{cVEMPs: ACS versus BCV Modes}

Via monaural ACS stimulation, all (100\%) right (control) necks showed clear cVEMPs, while all 10 left (lesion) necks revealed absent cVEMPs (Fig. 3). The mean threshold of ACS-cVEMP was $105 \pm 10 \mathrm{dBSPL}$, and the mean latencies of positive peak $\mathrm{I}$ and negative peak II, and peak-to-peak I-II amplitude were $5.93 \pm$ $0.40 \mathrm{~ms}, 7.41 \pm 0.52 \mathrm{~ms}$, and $5.4 \pm 1.5 \mu \mathrm{V}$, respectively (Table II).

In contrast, cVEMPs using BCV mode with the peak stimulus intensity of $139 \mathrm{dBFL}$ revealed $100 \%$ and $60 \%$ response rates in the right (control) and left (lesion) neck, respectively (Fig. 4). The mean latencies of positive peak I and negative peak II, and peak-to-peak I-II amplitude were $3.31 \pm 0.92 \mathrm{~ms}, 5.20 \pm 0.90 \mathrm{~ms}$, and $179 \pm$ $93 \mu \mathrm{V}$ for the control sides, and $3.48 \pm 0.72 \mathrm{~ms}, 5.25 \pm$ $0.77 \mathrm{~ms}$, and $134 \pm 36 \mu \mathrm{V}$ for the lesion sides, respectively. There was no significant difference between the two sides in this regard (Mann-Whitney $U$ test, $P>.05$, Table II). The mean thresholds of BCV-cVEMPs for the control and lesion sides were $132 \pm 4$ and $134 \pm 7$ dBFL, respectively, exhibiting nonsignificant difference $(P>.05)$.

\subsection{0uV \\ ACS-cVEMP}
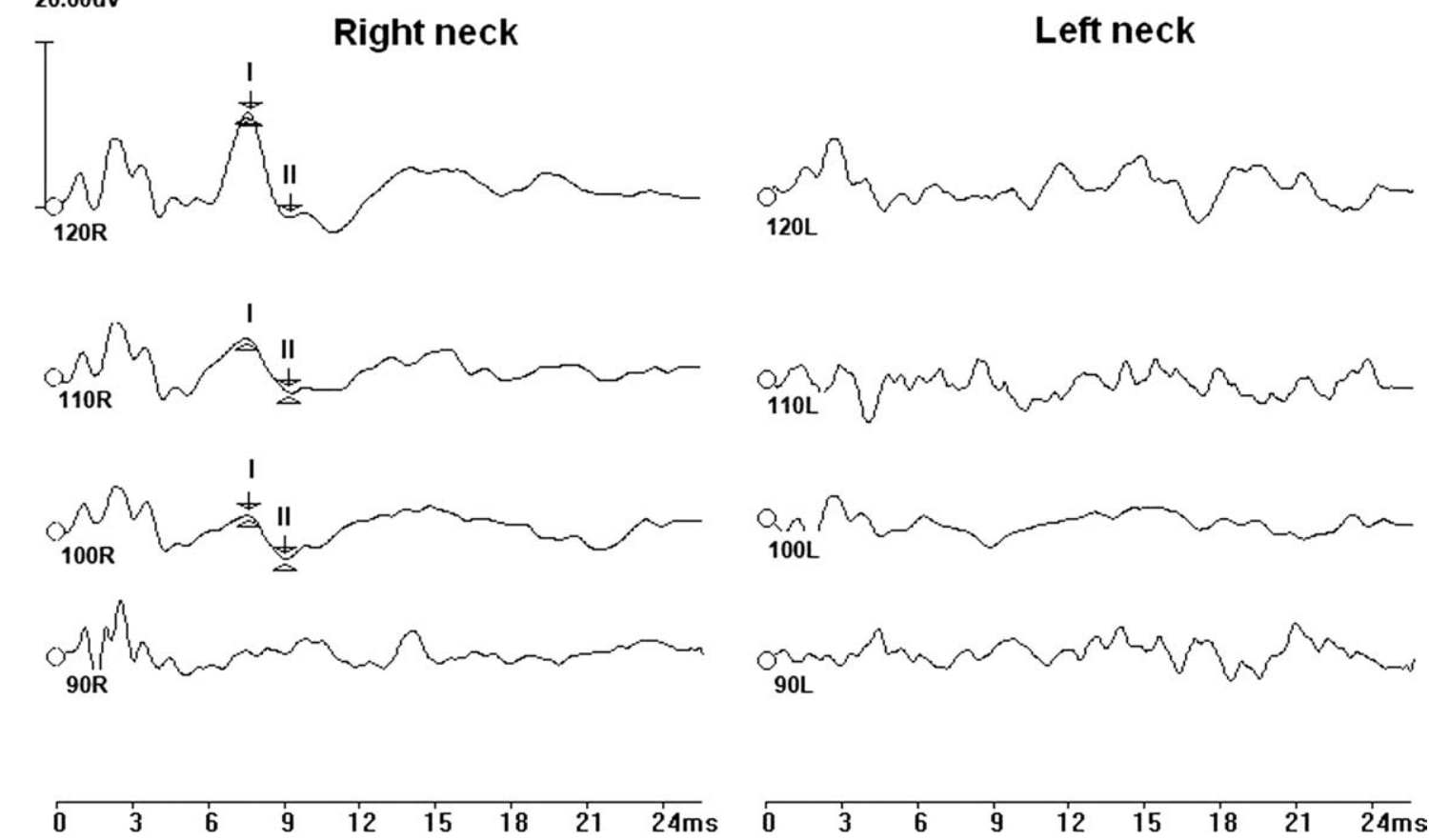

\section{\begin{tabular}{lllllllll}
\hline 0 & 3 & 6 & 9 & 12 & 15 & 18 & 21 & $24 \mathrm{~ms}$
\end{tabular}}

Fig. 3. Guinea pig treated with gentamicin (left), 1 week after operation. Via air-conducted sound monaural stimulation, clear cervical vestibular-evoked myogenic potentials are demonstrated on the right (control) neck, but not on the left (lesion) neck (120 = $120 \mathrm{dBSPL}$; = positive I; II = negative II). 
TABLE II.

Cervical Vestibular-Evoked Myogenic Potentials Using ACS Versus BCV Modes in Gentamicin-Treated Guinea Pigs.

\begin{tabular}{lcccccc}
\hline Stimulation Mode & Recording Neck Side & No. Ears & Prevalence, $\%$ & $\begin{array}{c}\text { Positive Peak } \\
\text { I Latency, ms }\end{array}$ & $\begin{array}{c}\text { Negative Peak } \\
\text { II Latency, ms }\end{array} \begin{array}{c}\text { Peak-to-Peak I-II } \\
\text { Amplitude, } \mu \text { V }\end{array}$ \\
\hline ACS & Right (control) & 10 & 100 & $5.93 \pm 0.40^{*}$ & $7.41 \pm 0.52^{*}$ & $5.4 \pm 1.5^{\star}$ \\
BCV & Left (lesion) & 10 & 0 & 0 & 0 & 0 \\
& Right (control) & 10 & 100 & $3.31 \pm 0.92^{*, \dagger}$ & $5.20 \pm 0.90^{*, \dagger}$ & $179 \pm 93^{*, \dagger}$ \\
& Left (lesion) & 10 & 60 & $3.48 \pm 0.72^{\dagger}$ & $5.25 \pm 0.77^{\dagger}$ & $134 \pm 36^{\dagger}$ \\
\hline \hline
\end{tabular}

Data are expressed as mean \pm standard deviation.

${ }^{\star} P<.001$, paired $t$ test.

${ }^{\dagger} P>.05$, Mann-Whitney $U$ test.

$\mathrm{ACS}=$ air-conducted sound; $\mathrm{BCV}=$ bone-conducted vibration

Comparing the characteristic parameters between ACS-cVEMPs and BCV-cVEMPs revealed significantly shorter latencies but larger amplitude for the BCVcVEMPs $(P<.001$, paired $t$ test, Table II).

\section{Morphological Study}

Morphological study was conducted using whole mount preparation of the utricle and saccule in the gentamicin-treated animals and observed under confocal microscope. The hair cells in the surface preparation were stained with rhodamine phalloidin, which revealed red hair cells and blue nuclei when counterstained with 4'-6-diamidino-2-phenylindole.

Grossly, both utricle (Fig. 5A, 5B) and saccule (Fig. $6 \mathrm{~A}, 6 \mathrm{~B})$ retained their contour either in the control or gentamicin-treated ears. In the utricular (Fig. 5C) and saccular macula (Fig. 6C) of the control ears, the hair cells with their well-recognized stereocilia were abundant and arranged in a regular order. In contrast with the lesion ears, substantial loss of hair cells was demonstrated in the utricular macula (Fig. 5D) and the saccular macula (Fig. 6D), with the stereocilia on top of some hair cells either fused or deformed.

\section{DISCUSSION}

Ocular VEMP has been suggested to reflect a crossed VOR in humans, ${ }^{3}$ which is originated from the saccular macula when using ACS stimulation, and from both utricular and saccular macula via BCV mode. ${ }^{11}$ Afferents from the saccular macula consisted of two parts, those from the hook region of the saccular macula course in the superior vestibular nerve and those from the elongated shank
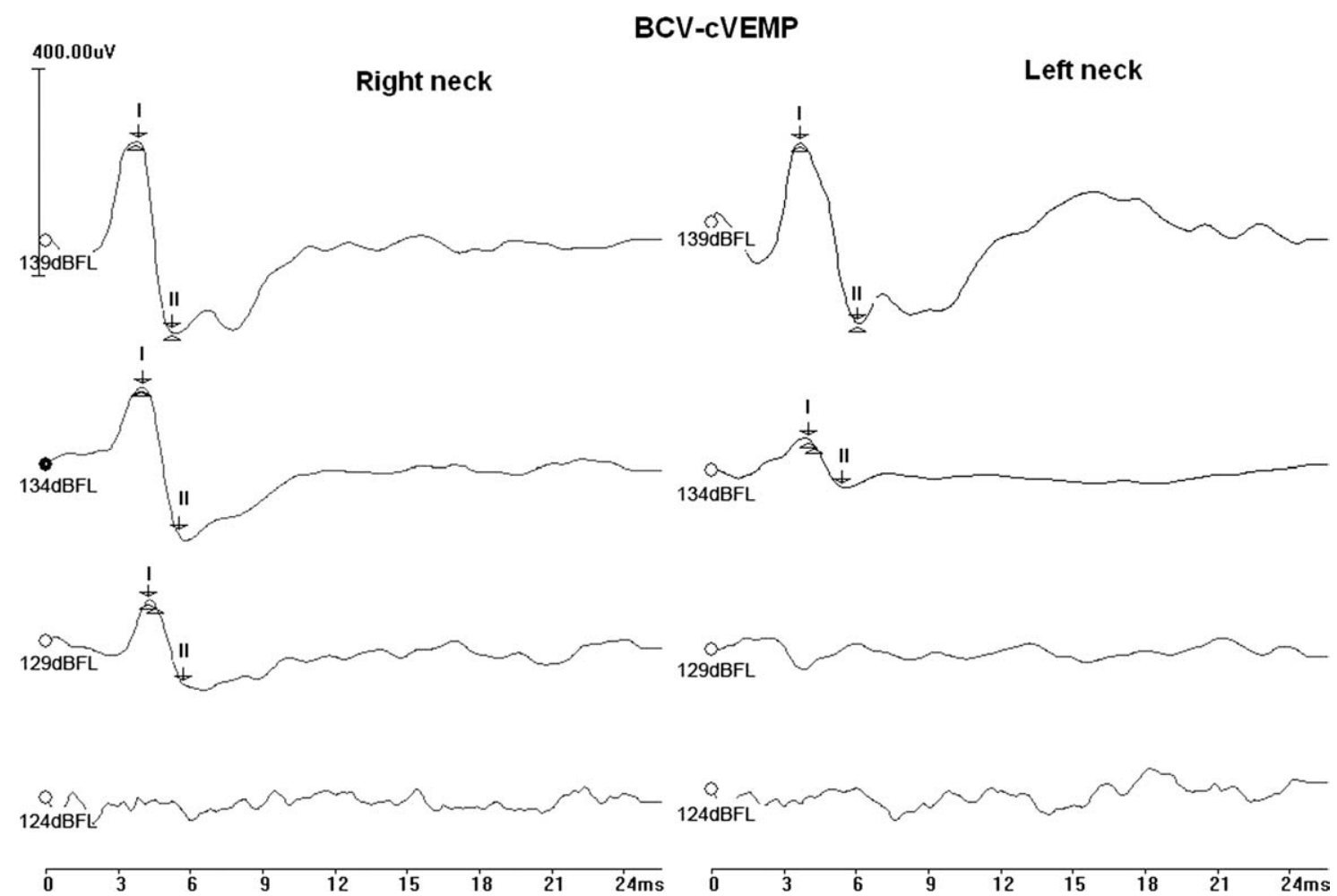

Fig. 4. Guinea pig treated with gentamicin (left), one week after treatment. Using bone-conducted vibration mode, clear cervical vestibularevoked myogenic potentials are demonstrated on the right (control) and left (lesion) neck (139 dBFL = $139 \mathrm{~dB}$ force level; I = positive I; II $=$ negative II). 

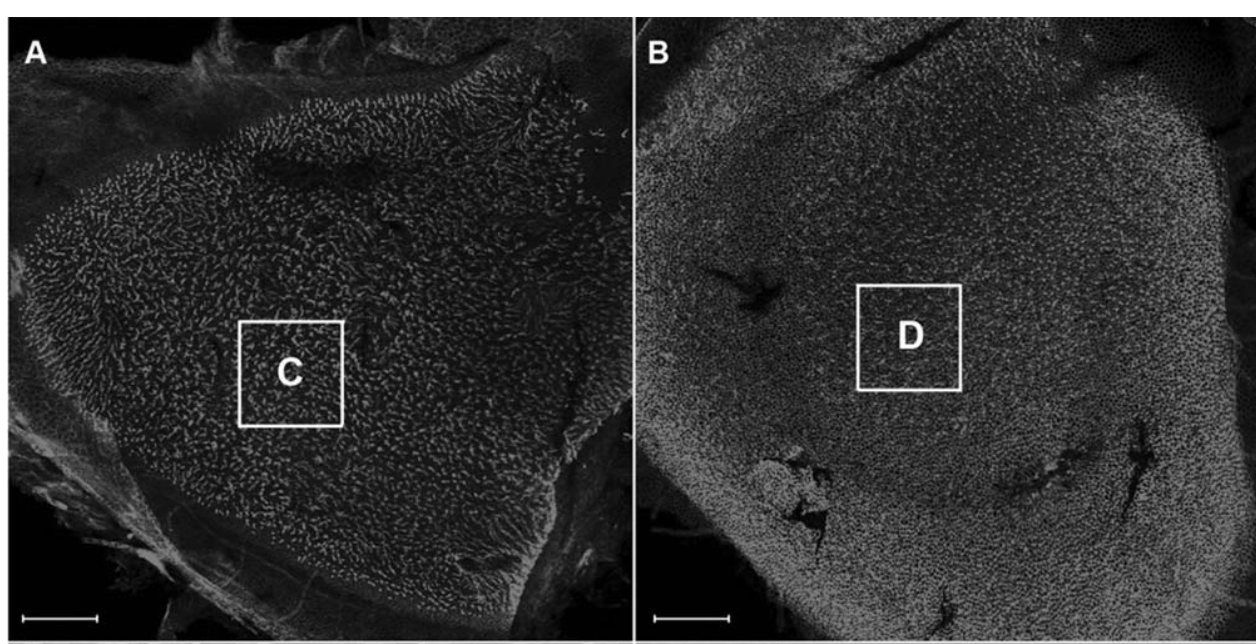

Fig. 5. Confocal images of the utricular surface of a gentamicin-treated guinea pig, one week after treatment. $(A, C)$ Control ear. $(B, D)$ Loss of hair cells of the utricular macula is noted in lesion ear, and the remaining hair cells scattered in disarray. The stereocilia on top of some hair cells are either fused or deformed. Bar $=10 \mu \mathrm{m}(\mathrm{A}, \mathrm{B})$ and $50 \mu \mathrm{m}(\mathrm{C}, \mathrm{D})$. C,D: magnification of boxes $C$ and $D$ from figures $A$ and $B$, respectively.
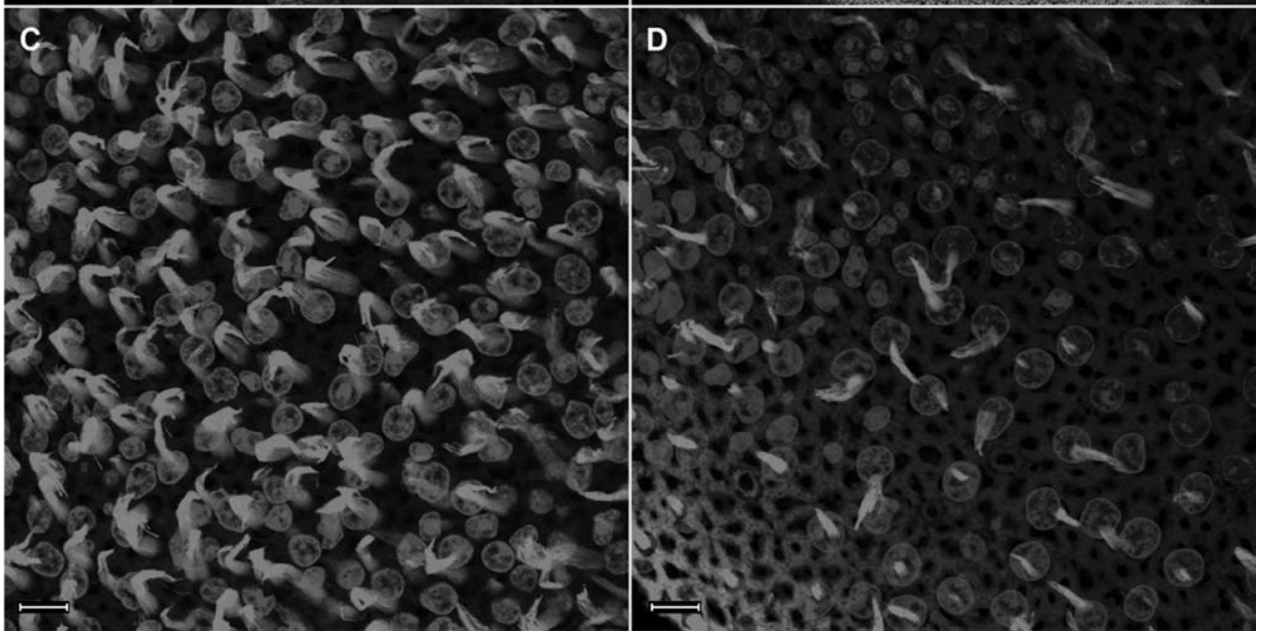

region travel in the inferior vestibular nerve. ${ }^{12,13}$ Restated, the superior vestibular nerve innervates the sensory epithelia of the horizontal and superior ampulla, the utricle, and a small part (hook region) of the saccular macula. In contrast, the inferior vestibular nerve supplies the main portion (shank region) of the saccular macula and the posterior ampulla.

Conventional instruments for evoked potentials are typically equipped with a maximum level of $120 \mathrm{~dB}$ pe SPL for air-conducted sound and $70 \mathrm{dBnHL}$ (107 dBFL) for boneconducted sound, which are sufficient for eliciting cVEMPs but not for oVEMPs (Fig. 1) because the normal thresholds for eliciting oVEMPs were $130 \pm 1 \mathrm{dBFL}$ in humans ${ }^{14}$ and $130 \pm 6 \mathrm{dBFL}$ in guinea pigs (this study).

Alternatively, a powerful vibrator with the stimulus intensity up to $139 \mathrm{dBFL}$ was utilized to trigger oVEMPs. Via BCV mode, all (100\%) gentamicin-treated animals showed absent oVEMPs on the control eyes, and clear oVEMPs on the lesion eyes (Fig. 2), which further supports that BCV mode is better than ACS mode in eliciting oVEMPs.

Unlike oVEMPs, cVEMPs via BCV stimuli demonstrated $100 \%$ response rates in the control sides, and $60 \%$ response rates in the lesion sides (Fig. 4; Table II). Because irregular afferents from both utricular and saccular macula are selectively stimulated at low stimulus intensities by BCV mode, ${ }^{4}$ could it be possible that utricular afferents contribute to the BCV-cVEMPs on the lesion side? From morphological studies in gentamicin-treated ears, both utricular and saccular macula were substantially damaged (Fig. 5 and Fig. 6), compatible with our previous reports. $^{8,9}$ Thus, ipsilateral utricular afferents downward affecting the cVEMPs can be neglected.

Next, because BCV stimuli delivering a repeatable tap onto the frontal bone, which may stimulate bilateral otolithic organs, simultaneously, thus, clear BCVcVEMPs in the lesion side are possibly contaminated from the responses of the opposite (control) side. The reason may be that $\mathrm{BCV}-\mathrm{cVEMPs}$ of the lesion side are likely to be responses arising from the opposite labyrinth following BCV stimulus conduction across the skull. ${ }^{14}$ Accordingly, if ACS mode is given monaurally, the crossed effect on ACS-cVEMP is minimal and can be negligible (Fig. 3). Conversely, when BCV stimuli were applied in guinea pigs, a crossed response may definitely contaminate the BCV-cVEMPs (Fig. 4). Thus, ACS mode is preferable to $\mathrm{BCV}$ mode in eliciting cVEMPs.

\section{Clinical Relevance}

In humans, when detecting cVEMPs via BCV mode, one cannot differentiate the lesion of saccular/inferior 

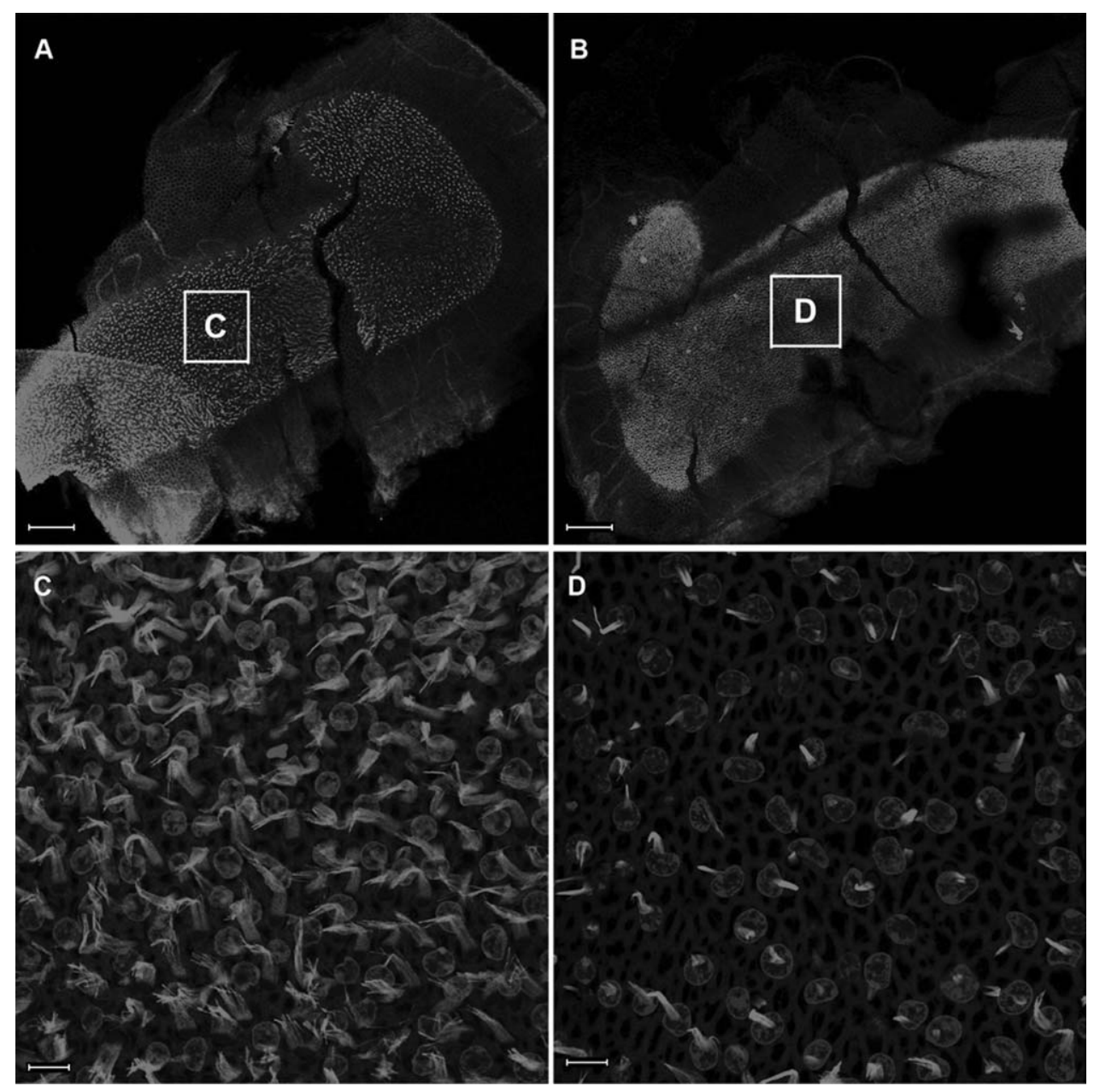

Fig. 6. Confocal images of the saccular surface of a gentamicin-treated guinea pig, one week after treatment. (A, C) Control ear. (B, D) Lesion ear demonstrates substantial loss of hair cells of the saccular macula. Bar $=10 \mu \mathrm{m}(\mathrm{A}, \mathrm{B})$ or 50 $\mu \mathrm{m}(\mathrm{C}, \mathrm{D})$.

vestibular nerve from that of utricular/superior vestibular nerve. This weakens the diagnostic value of the cVEMP test by BCV mode for localizing the site of inner ear pathology. ${ }^{5}$ Thus, the cVEMP test via ACS mode is specific for investigating the saccular disorder. With regard to the oVEMPs, ACS mode is not a reliable tool because it requires high-intensity, unpleasant, and possibly damaging sound intensities to elicit even an unreliable ACS-oVEMP. Conversely, oVEMPs can be elicited reliably by BCV stimuli at modest levels. ${ }^{6}$ Thus, the oVEMP test via BCV mode is preferable for investigating the utricular disorder. ${ }^{11}$ In guinea pigs, the appropriate animal models for cVEMPs and oVEMPs are also via ACS and BCV modes, respectively, which are consistent with those reported for humans. ${ }^{5}$

\section{CONCLUSION}

The cVEMP test via ACS mode is specific for investigating the saccular disorder, whereas the oVEMP test via $\mathrm{BCV}$ mode is preferable for investigating the utricular disorders in humans. The guinea pig model is consistent with the findings of humans. Restated, appropriate animal models for cVEMP and oVEMP in guinea pigs are via ACS and BCV modes, respectively.

\section{BIBLIOGRAPHY}

1. Welgampola MS, Myrie OA, Minor LB, Carey JP. Vestibular-evoked myogenic potential thresholds normalize on plugging superior canal dehiscence. Neurology 2008;70: 464-472.

2. Uchino Y, Sato H, Sasaki M, et al. Sacculocollic reflex arcs in cats. J Neurophysiol 1997;77:3003-3012.

3. Iwasaki S, McGarvie LA, Halmagyi GM, et al. Head taps evoke a crossed vestibulo-ocular reflex. Neurology 2007; 68:1227-1229.

4. Curthoys IS, Kim J, McPhedran SK, Camp AJ. Bone-conducted vibration selectively activates irregular primary otolithic vestibular neurons in the guinea pig. Exp Brain Res 2006;175:256-267.

5. Wang SJ, Weng WJ, Jaw FS, Young YH. Ocular and cervical vestibular evoked myogenic potentials: a study to determine whether air- or bone-conducted stimuli are optimal. Ear Hear 2010;31:283-288.

6. Cheng PW, Chen CC, Wang SJ, Young YH. Acoustic, mechanical and galvanic stimulation modes elicit ocular vestibular-evoked myogenic potentials. Clin Neurophysiol 2009;120:1841-1844.

7. Yang TH, Young YH. Click-evoked myogenic potentials recorded on alert guinea pigs. Hear Res 2005;205:277-283.

8. Day AS, Lue JH, Yang TH, Young YH. Effect of intratympanic application of aminoglycosides on click-evoked myogenic potentials in guinea pigs. Ear Hear 2007;28:18-25.

9. Lue JH, Day AS, Cheng PW, Young YH. Vestibular evoked myogenic potentials are heavily dependent on type I hair 
cell activity of the saccular macula in guinea pigs. Audiol Neurootol 2009;14:59-66.

10. Yang TH, Liu SH, Young YH. A novel inner ear monitoring system for evaluating ototoxicity of gentamicin eardrops in guinea pigs. Laryngoscope 2010;120:1220-1226.

11. Curthoys IS. A critical review of the neurophysiological evidence underlying clinical vestibular testing using sound, vibration and galvanic stimuli. Clin Neurophysiol 2010; 121:132-144.
12. De Burlet HM. Zur Innervation der Macula sacculi bei Saugetieren. Anat Anzig 1924;58:26-32.

13. Iwasaki S, Chihara Y, Smulders YE, et al. The role of the superior vestibular nerve in generating ocular vestibularevoked myogenic potentials to bone-conducted vibration at Fz. Clin Neurophysiol 2009;20:588-593.

14. Welgampola MS, Rosengren SM, Halmagyi GM, Colebatch JG. Vestibular activation by bone conduced sound. J Neurol Neurosurg Psychiatry 2003;74:771-778. 